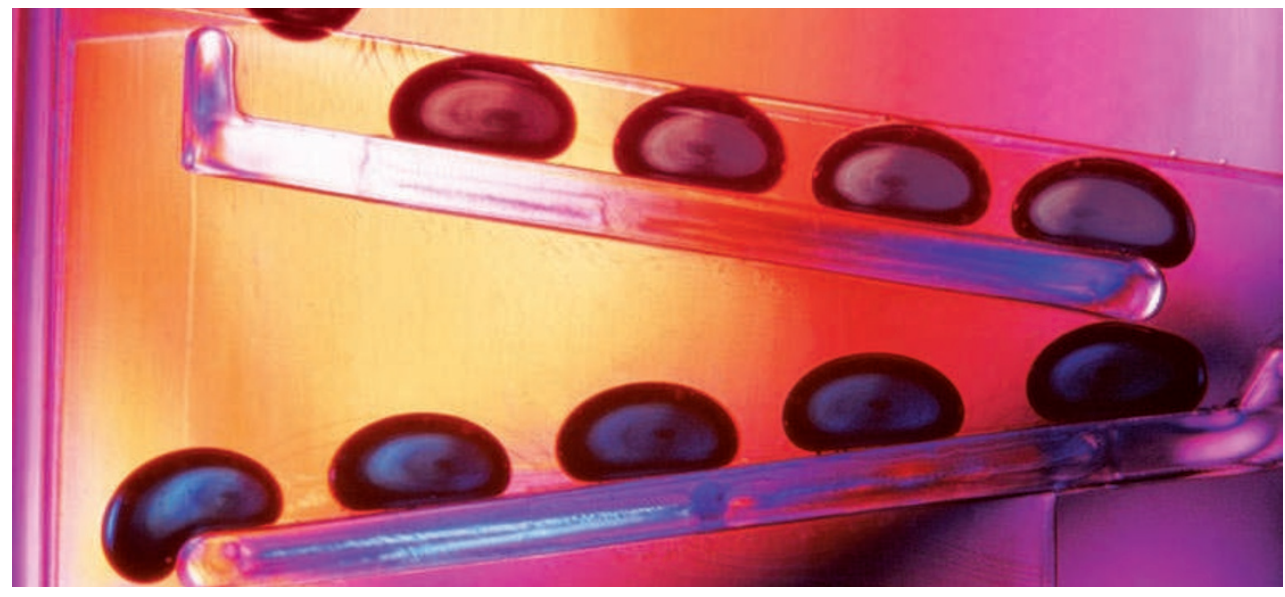

$\Rightarrow$ EPIGENETICS

\title{
Demethylation links cell fate and cancer
}

Genome-wide loss of DNA methylation is characteristic of many cancers; however, mutations in DNA methyltransferases (DNMTs) have not been linked with human tumours, so it has been unclear how this hypomethylation arises. New insights have been provided by a study in zebrafish showing that a tumour suppressor acts through a demethylase system to regulate cell fate decisions, and that loss of the normal control of demethylation contributes to tumorigenesis.

Previous studies have shown that adenoma formation after loss of the tumour suppressor gene adenomatous polyposis coli (APC) is reduced by inhibition of DNMT1. These findings suggest that APC may normally function to regulate levels of DNA methylation. To investigate how such regulation occurs, Rai and colleagues examined zebrafish embryos with homozygous apc mutations. Components of the DNA demethylase system were upregulated in this setting, accompanied by promoter hypomethylation of many genes involved in intestinal fate and colorectal cancer development. Knockdown of demethylase components restored normal levels of methylation. Together, these results suggest that the demethylase system is responsible for hypomethylation of target genes in involved in fate decisions, but that hypomethylation is prevented when APC is active. The authors also showed that this scenario is relevant to human cancer - APC-mutant colon adenomas showed upregulation of demethylase components and concomitant hypomethylation of a set of promoters that overlapped with those identified in zebrafish.

APC controls both Wnt signalling and retinoic acid (RA) biosynthesis; is one of these mechanisms responsible for the upregulation of demethylase components after APC loss? The authors treated apc-mutant embryos with RA or an inhibitor of $\beta$-catenin, a component of Wnt signalling. Dysregulation of Wnt signalling did not affect expression of demethylase components; however, RA treatment reversed the upregulation of the demethylase components induced by apc mutation, suggesting that RA is involved in regulation of DNA demethylase.

How does promoter demethylation of intestinal cell fate genes affect apc-mutant embryos? The authors showed that although these embryos can develop primordial intestines, which express key patterning genes, the intestinal cells do not differentiate. Knockdown of demethylase components led to increased intestinal differentiation, suggesting that demethylase functions to keep intestinal cells in an undifferentiated state after loss of Apc. Intriguingly, Rai et al. also observed increases in the levels of brain proliferation markers in apc mutants, suggesting that the demethylase system might have broader roles in determining developmental cell fates.

This study therefore provides important insights into the regulation of DNA methylation during development and in cancer, and highlights the role of DNA demethylation in this process. The authors suggest a model by which APC regulates a switch in DNA methylation dynamics - the demethylase system allows for plasticity and maintenance of progenitor cells, whereas RA and APC downregulate demethylase, leading to methylation of key developmental genes and commitment of progenitors to differentiation.

Meera Swami

ORIGINAL RESEARCH PAPER Rai, K. et al. DNA demethylase activity maintains intestinal cells in an undifferentiated state following loss of APC. Cell 142, 930-942 (2010) FURTHER READING Law, J. A. \& Jacobsen S. E.

Establishing, maintaining and modifying DNA methylation patterns in plants and animals. Nature Rev. Genet. 11, 204-220 (2010) 